\title{
Is an alcoholic fixative fluid used for manual liquid-based cytology accurate to perform HPV tests?
}

This article was published in the following Dove Press journal:

Pathology and Laboratory Medicine International

23 December 201I

Number of times this article has been viewed

\author{
Christian Garbar' \\ Corinne Mascaux' \\ Philippe De Graeve ${ }^{2}$ \\ Philippe Delvenne ${ }^{3}$ \\ 'Department of Biopathology, \\ Institute Jean Godinot, Reims Cedex, \\ France; ${ }^{2}$ Centre de Pathologie des \\ Coteaux, Toulouse, France; \\ ${ }^{3}$ Department of Pathology, University \\ of Liege, Tour de Pathologie, Domaine \\ Universitaire du Sart Tilman, Liège, \\ Belgium
}

\begin{abstract}
In Europe, the alternative centrifuge method of liquid-based cytology is widely used in cervical screening. Turbitec ${ }^{\circledR}$ (Labonord SAS, Templemars, France) is a centrifuge method of liquid-based cytology using an alcoholic fixative fluid, Easyfix ${ }^{\circledR}$ (Labonord). It is now well accepted that the association of liquid-based cytology and human papillomavirus test is indissociable of cervical screening. The aim of this work was to demonstrate that Easyfix alcoholic fluid is reliable to perform Hybrid Capture ${ }^{\circledR} 2$ (QIAGEN SAS, Courtaboeuf, France). In this study, 75 patients with colposcopy for cervical lesions served as gold standard. A sample was collected, at random, for Easyfix fixative cytological fluid and for Digene Cervical Sampler (QIAGEN). The results of Hybrid Capture 2 (with relative light unit $>1$ ) showed no statistical difference, a positive Spearman's correlation $(\mathrm{r}=0.82, P<0.0001)$, and a kappa value of 0.87 (excellent agreement) between the two fluids. It was concluded that Easyfix is accurate to use in human papillomavirus tests with Hybrid Capture 2.
\end{abstract}

Keywords: human papillomavirus, hybrid capture 2, Turbitec ${ }^{\circledR}$, cervix cytology, liquid-based cytology

\section{Introduction}

In Europe, the alternative method of manual liquid-based cytology (LBC) is widely used in cervical screening. ${ }^{1}$ The centrifuge method is the most common, for example the Turbitec ${ }^{\circledR}$ system (Labonord SAS, Templemars, France) of LBC, which uses a nonbuffered solution containing about $25 \%$ of ethylic alcohol and ethylenediaminetetraacetic acid (Easyfix ${ }^{\circledR}$; Labonord).

It is now well accepted that the association of LBC and human papillomavirus (HPV) test is indissociable of the screening for HPV-related cervical lesions, especially for atypical squamous cells of undetermined significance cytological lesions. ${ }^{2}$ One of the most common HPV tests is Hybrid Capture 2 (HC2; QIAGEN SAS, Courtaboeuf, France).

The aim of this work was to demonstrate that Easyfix fluid is technically reliable to use with $\mathrm{HC} 2$.

\section{Material and methods}

Colposcopies or conizations were performed on 75 women (age $35.7 \pm 10.8$ years). The final diagnosis was classified into four groups: within the normal limits (WNL) or no lesion $(n=13)$, cervical intraepithelial neoplasia grade $1(\mathrm{CIN} 1, n=6)$, grade 2 $(\mathrm{CIN} 2, \mathrm{n}=21)$, and grade $3(\mathrm{CIN} 3, \mathrm{n}=35)$. Colposcopy without biopsy but with cytology was performed in eight WNL that were negative for HPV and had no HPV
Department of Biopathology, Institute
Jean Godinot, I avenue General Koenig
BP I7I, 5 I 056 Reims Cedex, France
Tel +33 326504266
Fax +33 326504274
Email christian.garbar@reims.unicancer.fr

\footnotetext{
Pathology and Laboratory Medicine International 20I2:4 I-4

(C) 2012 Garbar et al, publisher and licensee Dove Medical Press Ltd. This is an Open Access article which permits unrestricted noncommercial use, provided the original work is properly cited.

Dovepress

http://dx.doi.org/| 0.2147/PLMI.S2803। 
history. The $61 \mathrm{CIN}$ patients and five other WNL patients had a biopsy.

At time of colposcopy, a cervix sample was collected, at random, for Easyfix fixative cytological fluid and for Digene Cervical Sampler (QIAGEN). Both fluids were sent to two different independent laboratories in France $(\mathrm{n}=54)$ and in Belgium $(n=21)$. HC2 assay on Cervical Sampler was made according to the manufacturer's instructions.

The HC2 assay on Easyfix was performed in $4 \mathrm{~mL}$ of residual Easyfix added to $400 \mu \mathrm{L}$ Sample Conversion Buffer (QIAGEN). The solution was pelleted at $4000 \mathrm{rpm}$ for 20 minutes. The supernatant was discarded. The cellular pellet was resuspended with $150 \mu \mathrm{L}$ denaturation solution (composed of Specimen Transport Medium ${ }^{\text {TM }}$ and Denaturation Reagent; QIAGEN).

Classical hybridization, detection, and calibration were made according to the HC2 kit instructions. Probes were used only against the high-risk HPV genotypes: 16, 18, 31, $33,35,39,45,51,52,56,58,59$, and 68 . The HC2 results were expressed as positive or negative, with relative light unit $\left(\right.$ RLU) $>1$ as a positive result. ${ }^{3}$

As negative controls, five samples each of Cervical Sampler and Easyfix alone were used. These were performed using the same HC2 methodology.

The results were expressed by percentage of observations or mean \pm standard deviation. The results of the colposcopy and biopsy served as gold standard.

Fisher's exact test, McNemar's test, Spearman's rank correlation, and Cohen's kappa test were performed. $P<0.05$ was considered significant. In general, a kappa value higher than 0.75 indicates excellent agreement. Analyse-it $^{\circledR} 2.22$ (Analyse-it Software Ltd, Leeds, United Kingdom) and Excel 2003 (Microsoft Corporation, Redmond, WA) were used for statistical analysis.

\section{Results}

A significant difference in age was observed between WLN (43.2 \pm 12.1 years) and CIN lesions $(\mathrm{CIN1}=30.2 \pm 4.0$ years; $\mathrm{CIN} 2=32.0 \pm 9.7$ years; CIN3 $=35.5 \pm 10.4$ years $)$.

All samples were fixed $13.1 \pm 13.9$ days before the HC2 test.

Table 1 shows HC2 results when RLU was $>1$. No statistical difference was found with McNemar's test, showing that the two methods were similar.

Three cases were discrepant: one false positive for Cervical Sampler in WLN (RLU 0.18 for Easyfix versus 19.7 for Cervical Sampler) and two false negatives - CIN2 and Cervical Sampler (RLU 318.8 versus 0.45); CIN3 and
Table I Comparison of human papillomavirus results for relative light unit $>$ I

\begin{tabular}{lll}
\hline & Cervical Sampler & Easyfix $^{\circledR}$ \\
\hline WLN & $1 / 13$ & $0 / 13$ \\
& $(7.6 \%)$ & $(0.0 \%)$ \\
CINI & $5 / 6$ & $5 / 6$ \\
& $(83.3 \%)$ & $(83.3 \%)$ \\
CIN2 & $20 / 21$ & $21 / 21$ \\
& $(95.2 \%)$ & $(100 \%)$ \\
CIN3 & $35 / 35$ & $34 / 35$ \\
& $(100 \%)$ & $(97.1 \%)$ \\
\hline
\end{tabular}

Notes: No statistical difference was observed. Easyfix ${ }^{\circledR}$ came from Labonord SAS (Templemars, France) and Cervical Sampler from QIAGENSAS (Courtaboeuf, France). Abbreviations: CIN, cervical intraepithelial neoplasia; WLN, within the normal limits or no lesion.

Easyfix (RLU 0.12 versus 15.32). It must be noted that RLU were always low, probably because samples had low viral concentration.

HC2 results when RLU was $>1$ showed a positive Spearman's correlation with $\mathrm{r}=0.82(P<0.0001)$. A kappa value of 0.87 showed excellent agreement between the two fluids.

Figures 1 and 2 show that RLU exponentially increased following patient groups: WLN, $1.6 \pm 5.2$ versus $0.18 \pm 0.04$ ( $P=$ no significance); CIN1, $17.9 \pm 71.5$ versus $182.7 \pm 314.8$ $(P=$ no significance $) ; C I N 2,215.3 \pm 468.2$ versus $484.3 \pm 684.9(P=0.004)$; and CIN3, $445.8 \pm 734.7$ versus $721.8 \pm 625.4(P=0.002)$ for Cervical Sampler and Easyfix, respectively. Thus, RLU of Easyfix increased faster than Cervical Sampler. Negative controls showed an RLU $0.24 \pm 0.14$ versus $0.60 \pm 0.21$ for Cervical Sampler and Easyfix, respectively (data not shown).

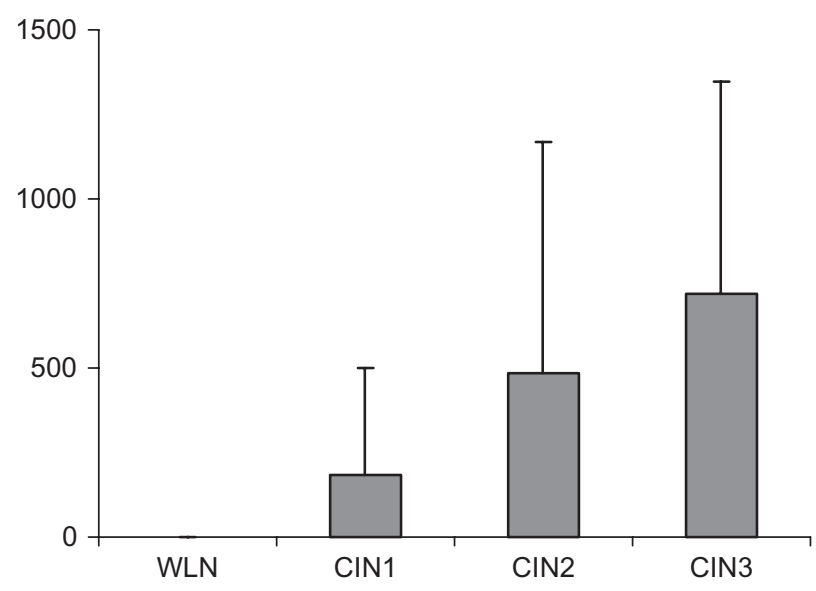

Figure I Easyfix ${ }^{\circledR}$ (Labonord SAS, Templemars, France): comparison of relative light units following colposcopic diagnosis.

Note: Relative light units exponentially increased following patients groups.

Abbreviations: CIN, cervical intraepithelial neoplasia; WLN, within the normal limits or no lesion. 


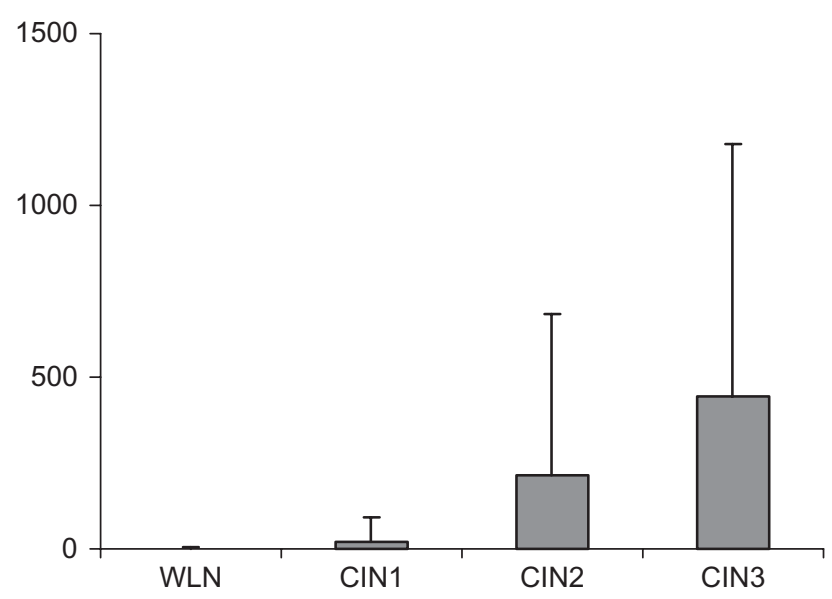

Figure 2 Cervical Sampler comparison of relative light units following colposcopic diagnosis.

Notes: Relative light units exponentially increased following patients groups. Relative light units for Cervical Sampler were lower than those for Easyfix ${ }^{\circledR}$. Abbreviations: CIN, cervical intraepithelial neoplasia; WLN, within the norma limits or no lesion.

\section{Discussion}

This work compared HPV/HC2 test using Easyfix cytological liquid fixative with Cervical Sampler. There was no statistical difference between these two fluids; Spearman's correlation was $r=0.85$ and a kappa value of 0.87 showed excellent agreement.

Curiously, HC2 values using Easyfix were higher than those with Cervical Sampler, likely due to difference in the sampling - the Rovers ${ }^{\circledR}$ Cervex-Brush ${ }^{\circledR}$ (Rovers Medical Devices BV, Oss, The Netherlands) gave more cells or HPV/ DNA in the Easyfix fluid. Both fluids showed a false positive (1/75 [1.3\%] for Cervical Sampler versus 0/75 for Easyfix) or false negative (1/75 [1.3\%] for Cervical Sampler versus $1 / / 75$ [1.3\%] for Easyfix) test. Interestingly, RLU were also lower than true results, likely corresponding with cases with lower DNA viral concentration. One case of CIN1 was both negative for Cervical Sampler and Easyfix, likely because HPV type was not tested by high-risk HC2 probes.

The aim of this study was to demonstrate the accuracy of Easyfix for HC2 method. Due to case selection, the results cannot be interpreted as an epidemiological study; therefore, results of sensitivity or specificity are not accurate.

In Europe, Easyfix is widely used with the low-cost liquid-based method Turbitec. Turbitec is an alternative method to automated and more expensive LBC methods. In 2000, Johnson et $\mathrm{al}^{4}$ described a similar method of LBC using a Hettich cytocentrifuge (Andreas Hettich $\mathrm{GmbH}$, Tuttlingen, Germany). Garbar et $\mathrm{al}^{5}$ was the first to demonstrate that Turbitec LBC had the same efficiency of LBC methods approved by United States Food and Drug Administration. More recently, Van Hemel et $\mathrm{al}^{6}$ demonstrated the efficiency of Turbitec in 632 colposcopies. It is now well accepted that the association of LBC and HPV test is indissociable of the screening of HPV-related cervical lesions, especially for atypical squamous cells of undetermined significance cytological lesions. ${ }^{2}$ The adequacy of fixative fluid to perform $\mathrm{HPV}$ tests is thus vital.

In 2004, Leduc et al, ${ }^{7}$ with a comparative study of Cervical Sampler in 256 samples, were the first to demonstrate the feasibility of performing $\mathrm{HC} 2$ with Easyfix. They found a kappa value of 0.76 ; lower than the kappa value in the present study. However, it must be noted that the methodology of sample preparation for $\mathrm{HC} 2$ was not the same in both studies, ie, $1.5 \mathrm{~mL}$ of Easyfix versus $4 \mathrm{~mL}$ used in the present study.

In 2005, a comparative study between HC2 and a polymerase chain reaction (PCR) technique, using QIAamp DNA Blood Mini Kit (QIAGEN) in 72 samples fixed with Easyfix, showed a kappa value of 0.89 , similar to the present results. ${ }^{4}$ Interestingly, even after $3.2 \pm 0.9$ months at room temperature the stability of DNA tested by beta globin was excellent.

INNO-LiPA HPV Genotyping Extra (Innogenetics NV, Gent, Belgium) is a new promising method that could be applied on cervical cell specimens. ${ }^{8}$ In 2007, Fontaine et al ${ }^{9}$ compared PCR sequencing, INNO-LiPA, and HC2 in 162 samples fixed with Easyfix. They demonstrated the stability of HPV-DNA for the three different methods (HC2 and INNO-LiPA: kappa value 0.552; HC2 and PCR: kappa value 0.729 ; PCR and INNO-LiPA: kappa value 0.843 ), with kappa values similar to the present study.

\section{Conclusion}

The results demonstrated the stability of DNA in Easyfix alcoholic cytological fixative fluid and its accuracy in HPV tests with $\mathrm{HC} 2$.

\section{Disclosure}

The authors report no conflicts of interest in this work. None of the authors has any financial involvement with Labonord. Labonord provided only disposable supplies for this study.

\section{References}

1. Cochand-Priollet B, Fabre M. Cytopathologie gynécologique en milieu liquide. Paris: Elsevier SAS; 2003.

2. Jordan J, Arbyn M, Martin-Hirsh P, et al. European guidelines for quality assurance in cervical cancer screening: recommendations for clinical management of abnormal cervical cytology, part 1. Cytopathology. 2008; 19(6):342-354.

3. Rijkaart DC, Coupe VM, van Kemenade FJ, et al. Comparison of Hybrid Capture 2 testing at different thresholds with cytology as primary cervical screening test. Br J Cancer. 2010;103(7):939-946. 
4. Johnson T, Maksem JA, Belsheim BL, Roose EB, Klock LA, Eatwell L. Liquid-based cervical-cell collection with brushes and wooden spatulas: a comparison of 100 conventional smears from high-risk women to liquid-fixed cytocentrifuge slides, demonstrating a cost-effective, alternative monolayer slide preparation method. Diagn Cytopathol. 2000; 22(2):86-91.

5. Garbar C, Mascaux C, Fontaine V. Efficiency of an inexpensive liquidbased cytology performed by cytocentrifugations: a comparative study using histology as reference standard. Cytojournal. 2005;2:15-22.

6. Van Hemel BM, Buikema HJ, Groen H, Suurmeijer AJ. Accuracy of low priced liquid-based method for cervical cytology in 632 women referred for colposcopy after a positive Pap smear. Diagn Cytopathol. 2009;37(8):579-583.
7. Leduc F, Bonniere M, Farre I, Staelen P. Human papillomavirus detection by hybrid capture II assay with the liquid cell preservation solution Easyfix. Ann Biol Clin (Paris). 2004;62(6):155-162. French.

8. Galan-Sanchez F, Hernandez-Menendez M, De Los Rios Hernandez MA, Rodriguez-Iglesias M. Performance of the new INNO-LiPA HPV extra to genotype human papillomavirus in cervical cell specimens. Acta Cytol. 2011;55(4):341-343.

9. Fontaine V, Mascaux C, Weyn C, et al. Evaluation of combined general primer-mediated PCR sequencing and type-specific PCR strategies for determination of human papillomavirus genotypes in cervical cell specimens. J Clin Microbiol. 2007;45(3):928-934.

\section{Publish your work in this journal}

Pathology and Laboratory Medicine International is a peer-reviewed, open access journal focusing on innovative basic research and translational research related to pathology or human disease. The journal includes original research, updates, case reports, reviews and commentaries on current controversies. The Academic Sponsor of this journal is the Chinese American Pathology Association (CAPA). The manuscript management system is completely online and includes a very quick and fair peer-review system. Visit http://www.dovepress.com/testimonials.php to read real quotes from published authors.

Submit your manuscript here: http://www.dovepress.com/pathology-and-laboratory-medicine-international-journal 